\title{
Innovando en el aula de Educación Primaria con GT 6
}

\section{Article Innovation in Primary Education classroom with GT 6}

\author{
Verónica Marín Díaz ${ }^{1}$, Begoña E. Sampedro-Requena ${ }^{2}$ \\ ${ }^{1}$ Departamento Educación, Universidad de Córdoba, España (vmarin@uco.es) \\ ${ }^{2}$ Departamento Educación, Universidad de Córdoba, España (bsampedro@uco.es)
}

Recibido el 26 de noviembre de 2015; revisado el 1 de marzo de 2016; aceptado el 4 de marzo de 2016; publicado el 1 de junio de 2016

\section{RESUMEN:}

Trabajar hoy en la esfera educativa supone estar a la vanguardia de todas las necesidades, recursos, demandas y ofertas que la sociedad lanza al sistema educativo. Una de esas demandas es la formación tecnológica de los estudiantes. Para ello los docentes emplean un gran número de herramientas que hagan atractivo no solo el currículo sino también la vida en las aulas. En ese espacio los videojuegos se convierten en un recurso que permite acercar la realidad social a los estudiantes, sacando la enseñanza de las aulas tradicionales a las digitales. En las siguientes páginas presentamos una propuesta de innovación docente basada en un videojuego no educativo, Gran Turismo 6. Se presentan una batería de actividades centradas en varias áreas: Ciencias Sociales y Educación Física, así como los contenidos que se quieren enseñar.

\section{PALABRAS CLAVE: VIDEOJUEGO, EDUCACIÓN PRIMARIA, ESTUDIANTE, INNOVACIÓN.}

\begin{abstract}
:
Work today in the educational sector means being at the vanguard of the needs, resources, demand and supplys that society throws the education system. One of these demands is the technological training of students. For this teachers must use a large number of tools, that make it more attractive not only on a curriculum but also for life in the classroom. In that way space video games become a recourse that can bring social reality to students, changing the traditional class teaching to digital - In the next pages we present an innovation proposal
\end{abstract}

based on non-educational video game, Gran Turismo 6. It presents a large number of activities focused in several areas: Social Sciences and Physical Education are presented, and also the content that you want to teach.

\section{KEYWORDS: VIDEOGAME, EDUCATION, STUDENT, INNOVATION.}

\section{INTRODUCCIÓN}

El crecimiento tecnológico de la sociedad implica hoy un cambio en la forma de mirar la participación de los individuos en el crecimiento y devenir de esta. Como ya han señalado algunos autores (Marín, 2014) todas las áreas en las que el sujeto se desarrolla están o se encuentran imbricadas a las tecnologías de la información y la comunicación (TIC), siendo quizás la más afectada la educativa, dado el papel vertebrador que juega en el proceso de socialización de las personas.

Centrando el eje de atención en el ámbito académico, compartimos con Munyoka, Kadymatimba, Manzirra, Tutoni, Chibonda y Madzwamuse (2014) que los estudiantes que hoy pueblan las aulas de los centros escolares de cualquier nivel educativo, viven en la denominada era digital, la cual ha promovido un aprendizaje de carácter creativo, autónomo y flexible, aspectos estos que las TIC ofrecen. Esta alfabetización conlleva tanto el logro como el desarrollo de las competencias básicas que se demandan a los estudiantes hoy (Gutiérrez, 2008). 
Al igual que Lari, Näykki y Jävelä (2012), consideramos que las TIC son el mecanismo más significativo de transformación del proceso de aprendizaje, ya que permite la creación de nuevos escenarios formativos en los que se produce de forma constante la información y se potencia la creación de conocimiento. Es por ello que consideramos que la formación ha de incorporar este tipo de recursos y perspectivas en los procesos de enseñanza y aprendizaje.

En estas líneas que a continuación desgranaremos vamos a exponer una propuesta de acción docente en las aulas de Educación Primaria a través de las TIC en general y, dentro de ellas, nos centraremos en concreto en los videojuegos, pero no en aquellos que han sido catalogados como educativos o "serius games".

Presentaremos, a continuación, una propuesta de trabajo interdisciplinar que permitirá al docente, a través de un videojuego convencional, alcanzar el desarrollo de una serie de contenidos curriculares en la etapa de educación primaria.

\subsection{Los videojuegos en el ámbito educativo}

El paso por diversas revoluciones ha provocado que la sociedad actual viva un momento tecnológico sin precedentes (Bidarian, Bidarian y Davoudi, 2011). Bajo este paraguas digital las diversas herramientas que se han ido creando han ido abriéndose un hueco en las diferentes áreas de la vida de los individuos; de este modo es habitual hablar hoy de sociedad 2.0. Dentro de la misma, y poniendo nuestra atención en la educación, son varios los autores que han incorporado la expresión 2.0 a dicho ámbito, así bien podemos hablar de educación 2.0, escuela 2.0 o currículo 2.0 (Cabero, 2009; Danciu y Grosseck, 2011). Igualmente encontramos que el abanico de recursos digitales creados por y para la formación de los sujetos ha ido creciendo día a día de forma vertiginosa, basta con mirar la biblioteca digital de la Fundación Germán Sainz de Ruiz Pérez (http://www.fundaciongsr.com/) para constatar esta circunstancia; por ello debemos ser conscientes y no olvidar que bajo el paraguas de Internet se crean, modifican y mueren habitualmente más de 3000 herramientas. No obstante, podemos cuestionarnos si todas las creadas son idóneas para el desarrollo del currículo educativo.

Es obvio, que si solo centramos nuestro eje de atención en los creados exclusivamente para el escenario formativo, andaremos errados en la consecución de los logros académicos, puesto que el docente, de manera reiterada, que no intencionada, emplea un número concreto de ellos, los cuales acaban convirtiéndose en una cotidianeidad para el estudiante, pero si optamos por emplear recursos que originalmente no fueron pensados para ser empleados en las aulas, será fácil caer en la necesidad de justificar la idoneidad de estos como herramientas que potencien los procesos de enseñanza y aprendizaje. En esta situación se encuentran los videojuegos; concebidos en su origen para entretener y divertir, alejados de una esfera formativa, poco a poco se han ido incorporando a la dinámica del profesorado durante el acto educativo. Prueba de ello es el considerable aumento de docentes que ya en 2012, según la Asociación Española de Videojuegos (ADEVI, anteriormente denominada aDeSe) (http://www.aevi.org.es/) los habían incorporado a su elenco de recursos metodológicos y como corrobora el trabajo de García, Cortés y Lacasa (2014).

Si hacemos un poco de historia, en el momento en que aparecieron los videojuegos el consumo de televisión por parte de niños y adolescentes descendió considerablemente, dado que permitía una interacción y un feedback continuo con el contenido de la pantalla, presentaban una gran carga audiovisual, haciendo asimismo más llamativa la pantalla en la que se debía centrar la atención, así como un gran dinamismo, cosa que la televisión, reina de la esfera lúdica, no ofrecía. Los diversos avatares de la vida hicieron que estos pasaran por etapas de gran auge (años 80) y de un gran declive (años 90); hoy nos encontramos ante otro gran momento de esplendor, como así lo reflejan el número de unidades vendidas tanto en Europa como en España (Marín, 2015; Marín. López y Maldonado, 2015).

Al ser un elemento que se encuentra totalmente imbricado en la vida de los niños, adolescentes y jóvenes (Martín, 2015), debemos cuestionarnos si cabe la posibilidad de emplear estos dentro de las cuatro paredes del aula. Desde nuestra perspectiva consideramos que han de ser vistos y sentidos como una herramienta al servicio del desarrollo curricular, puesto que facilita al docente encontrar diversos materiales educativos además de potenciar la interacción de la realidad escolar con la social o personal. Así el estudio realizado por Cuenca, Martín y Estepa (2014), señala que el profesorado en formación inicial consultado considera que el 16\% puede ser empleado para enseñar contenidos matemáticos, el $12.8 \%$ y el $16.6 \%$ relativos a ciencias naturales y sociales respectivamente, un 9.6\% piensa que se puede emplear en el aula de lengua, mientras que el $7.4 \%$ en artes plásticas; por último, un $1 \%$ opina que servirían para desarrollar el contenido curricular del área de educación física, 
idioma y música. En esta línea se puede encontrar el trabajo de Kebritchi, Hirumi y Bai (2010), quienes comprobaron que el empleo de los videojuegos potenciaba el aprendizaje de los conocimientos vinculados al álgebra.

Podemos decir que los videojuegos tienen tanto aspectos positivos como negativos, que pueden hacer que la cuestión anteriormente señalada se decante hacia su uso o su rechazo. En lo que se refiere a los primeros podemos indicar que estos son:

- Potenciar la curiosidad por aprender.

- Favorecer determinadas habilidades, como las motrices (ojo-mano).

- Permite el desarrollo transversal de las diversas áreas del currículo.

- Refuerza la autoestima, puesto que el valor de uno mismo se ve potenciado.

- Refuerza la capacidad de atención y la memoria.

- Desarrolla el pensamiento reflexivo y el razonamiento.

- Aumenta la visión y la perspectiva con la que se plantea el trabajo colaborativo y cooperativo.

- Aumenta la capacidad de relación (socialización), la capacidad visual y la espacial.

- Desarrolla el vocabulario (identificación de conceptos y significados).

- Desarrolla la capacidad lógico-matemática.

- Desarrolla conductas socialmente aceptadas.

- Ayuda a disminuir conductas impulsivas y/o de autodestrucción, de ansiedad y/o de estrés.

- Permite el tratamiento de algunas minusvalías.

- Enfatizan la resolución de conflictos.

- Fomenta el meta-análisis.

- Apoya el reconocimiento inductivo (Contreras, 2013; Marín, 2012a; Marín, 2014; Blumberg y Randall, 2015).

Con respecto a los segundos, podemos señalar:

- Creación de pautas de conducta antisociales.

- Empleo de temáticas con una gran carga de agresividad, dado que esta se presentan de forma continuada a través de la aparición de monstruos, alienígenas o extraterrestres, zombis, peleas callejeras, combates reglados sangrientos, asesinatos indiscriminados, atropellos, etc.

- Atentar contra la salud. Provoca tensión ocular, cambios en la circulación sanguínea, aumento de la frecuencia cardíaca y de la presión arterial, epilepsia, etc.

- Tiene una naturaleza adictiva.
- Trasgresión de las normas de la física.

- Bloqueo de la mente.

- Incapacidad para desarrollar otro tipo de actividades tanto lúdicas como educativas.

- Presentar un gran número de ellos sonidos estridentes y molestos.

- Excesivo tiempo de juego.

- Falta de sueño.

- El papel de la mujer está en un segundo plano.

- Ínfimo valor a la vida humana.

- El fin último del juego es llegar a la meta, cueste lo que cueste (Marín, 2012a; Contreras, 2013; Engelhardt y Mazurek, 2014; Marín, 2014; Blumberg y Randall, 2015).

Como vemos, la existencia de argumentos a favor $\mathrm{y}$ en contra va a determinar que el docente se aventure en su empleo. A nuestro juicio, la incorporación de un elemento de la vida social de los estudiantes en su espacio dedicado a la educación formal va a implicar que el nivel de atención de los estudiantes sea mayor, puesto que no se puede determinar con certeza, o establecer una relación casual entre el empleo de videojuegos no educativos y el desarrollo de conductas disruptivas (Moncada y Chacón, 2012). Por otra parte el estudio llevado a cabo por Interactive Software Dederation of Europe (ISFE) para aDeSe y denominado Gametrack: The Videogames in Europe Consumer Study, refleja que el $47 \%$ y el $25 \%$ de los europeos considera que los videojuegos benefician las habilidades creativas y sociales de los más jóvenes.

En el mercado existen diversos tipos de videojuegos; en este artículo nos hemos detenido en la elaborada por Marqués en 2007; estos pueden ser vinculados a la educación a través de diferentes objetivos, atendiendo a la siguiente clasificación podemos establecer la relación entre ambos elementos:

- ARCADE: potencian el desarrollo psicomotor y la orientación espacial.

- DEPORTES: permite de nuevo desarrollar habilidades psicomotoras y el conocimiento de las reglas y estereotipos propios del deporte.

- AVENTURA Y ROL: promueve el desarrollo del conocimiento de diferentes temáticas, aportando valores y contravalores.

- SIMULADORES: permite aprender a controlar la tensión y desarrollar la imaginación. 
- ESTRATEGIA: permite aprender a administrar los recursos que suelen ser escasos.

- JUEGOS DE LÓGICA: desarrollan la lógica, la percepción espacial, la imaginación y la creatividad.

- DE PREGUNTAS: repasar lecciones del currículo.

La utilización de los videojuegos y juegos digitales a nivel educativo debe partir de la relación entre el mensaje que estos emiten y el universo que recrean y que el jugador, además, debe saber interpretar, pero he ahí un hándicap, a veces dicho universo no es bien interpretado, bien por no conocer el código icónico bien por no conocer el sentido del mensaje en sí mismo (Marín, 2012b).

\section{GT 6 EN EL AULA DE EDUCACIÓN PRIMARIA}

El videojuego de carreras de coches o de simulación de carreras Gran Turismo 6 o como se le conoce GT6 (http://www.gran-turismo.com/es/) fue lanzado al mercado para conmemorar los 15 años de su primer lanzamiento en diciembre de 2013 por la empresa Sony, creado para su consola Play Station 3. Esta nueva edición del juego conlleva nuevos circuitos, como es el de Ascari en Ronda (Málaga España) y nuevos vehículos como el Alfa Romeo TZ3 Stradale, el BMW Z4, el Ferrari XX4 o el McLaren F1 GTR Race Car Base Model. Inicialmente creado para dos jugadores, desde 2014 se puede ampliar el cupo hasta 16 en su modalidad online. La mayor crítica negativa que ha recibido esta saga ha sido la poca versatilidad de sus actualizaciones; no obstante, las positivas han girado en torno al aumento del número de jugadores, así como la gran gama de vehículos y circuitos disponibles.

Dado que el juego se desarrolla a lo ancho y largo del planeta permite al docente introducir al estudiante en los conocimientos del área de Educación física, dado que deberá ser consciente de la preparación física que debe tener un piloto profesional. Por otra parte, si nos centramos en el área de Ciencias sociales, el estudiante se podrá centrar en encontrar información sobre país del circuito seleccionado. Así por ejemplo, si se elige el circuito de la ciudad de Ronda, el docente le podrá enseñar la especial climatología que tiene la provincia de Málaga, así como la cultura de la Comunidad Autónoma Andaluza, en la que está ubicada la localidad, las plantas, árboles y vegetación en general de la zona, las culturas residentes, etc.

\section{Acciones previas a la utilización del juego en el aula:}

Una vez determinado el momento curricular en que se va a implementar el videojuego en el aula, es necesario realizar una batería de actividades de carácter previo, que de un lado ayuden al docente a determinar los conocimientos previos que tienen los estudiantes sobre el tema que se pretende trabajar, y de otro y en este caso, de los intereses del alumnado hacia este tema.

Así desde aquí se proponen las siguientes:

1. Debate: presentación del código de la circulación.

2. Google maps: localización geográfica circuitos. Búsqueda de vocabulario.

3. Visionado de una carrera real: establecer una relación entre las normas del código de circulación y la acción de la carrera.

Atendiendo al área de Educación Física, los objetivos que pretendemos cubrir son:

A. Conocer y valorar su cuerpo y la actividad física como medio de exploración y disfrute de sus posibilidades motrices, de relación con los demás y como recurso para organizar el tiempo libre.

B. Apreciar la actividad física para el bienestar, manifestando una actitud responsable hacia uno mismo y las demás personas y reconociendo los efectos del ejercicio físico, de la higiene, de la alimentación y de los hábitos posturales sobre la salud.

C. Utilizar sus capacidades físicas, habilidades motrices y su conocimiento de la estructura y funcionamiento del cuerpo para adaptar el movimiento a las circunstancias $y$ condiciones de cada situación.

D. Adquirir, elegir y aplicar principios y reglas para resolver problemas motores y actuar de forma eficaz y autónoma en la práctica de actividades físicas, deportivas y artísticoexpresivas.

E. Regular y dosificar el esfuerzo, llegando a un nivel de auto-exigencia acorde con sus posibilidades y la naturaleza de la tarea.

F. Utilizar los recursos expresivos del cuerpo y el movimiento, de forma estética y creativa, comunicando sensaciones, emociones $\mathrm{e}$ ideas.

Y del área de Ciencias sociales serían:

- Identificar los principales elementos del entorno natural, social y cultural, analizando su organización, sus características e 
interacciones y progresando en el dominio de ámbitos espaciales cada vez más complejos.

- Comportarse de acuerdo con los hábitos de salud y cuidado personal que se derivan del conocimiento del cuerpo humano, mostrando una actitud de aceptación y respeto por las diferencias individuales (edad, sexo, características físicas, personalidad).

- Reconocer y apreciar la pertenencia a grupos sociales y culturales con características propias, valorando las diferencias con otros grupos y la necesidad del respeto a los derechos humanos.

- Reconocer en el medio natural, social y cultural, cambios y transformaciones relacionados con el paso del tiempo e indagar algunas relaciones de simultaneidad y sucesión para aplicar estos conocimientos a la comprensión de otros momentos históricos.

Las competencias en las que se centrará nuestra atención serán: matemática, conocimiento y la interacción con el mundo físico; tratamiento de la información y digital y autonomía e iniciativa personal (LOMCE, 2013).

Centrándonos en los contenidos, en lo que se refiere al área de Educación Física organizados estos en función de los bloques temáticos establecidos para este nivel educativo se trabajaran.
Bloque 1. El entorno y su conservación

- Exploración de distancias, recorridos y situaciones de objetos y personas en relación con el espacio.

- Determinar costes de construcción de un circuito, tanto a nivel medio ambiental como económico. social

Bloque 4. Personas, culturas y organización

- Búsqueda e identificación de las diferentes culturas del país que acoge la construcción del circuito (historia, renta per cápita, religión, etc.)

Con respecto al área de Ciencias sociales: Bloque 1. El cuerpo, imagen y percepción

- Dibujar su propio cuerpo. Bloque 2. Habilidades motrices

- Jugar una partida al volante de un coche de carreras, y marcar en la plantilla diseñada, los logros y dificultades que su cuerpo experimenta durante la conducción.

Bloque 3: Actividades artístico-deportivas

- Después de participar en una carrera en el juego indicar sus sensaciones, emociones y sentimientos a través del cuerpo.

Una vez realizadas estas actividades se procederá a evaluar a través de una rúbrica (ver tabla 1).

Tabla 1: Rúbrica de evaluación

\begin{tabular}{ccc} 
Área & Elementos de evaluación & $\begin{array}{c}\text { Puntuación } \\
(0-100)\end{array}$ \\
\hline Educación Física & $\begin{array}{c}\text { Es capaz de dibujar las partes de su cuerpo y diferenciarlas } \\
\text { Sabe determinar las dificultades de una exposición máxima } \\
\text { de tensión muscular }\end{array}$ \\
& Sabe reconocer los peligros de una conducción extrema \\
$\begin{array}{c}\text { Conocimiento del área } \\
\text { natural, social y } \\
\text { cultural }\end{array}$ & Reconoce la realidad cultural de la zona donde está el \\
circuito
\end{tabular}

El empleo de una rúbrica de evaluación permitirá al docente concretar los aspectos logrados, más allá de las actividades llevadas a cabo a raíz de emplear la videoconsola. En este caso aportarán datos cualitativos para determinar el avance en la consecución de las competencias anteriormente señaladas que se iban a trabajar siendo en principio, en este caso, un mero elemento orientativo.

\section{REFLEXIÓN FINAL}

El sistema de enseñanza tradicional tenía tres problemas graves según, señalaba Fandos (2006) geográficos, temporales y de demanda-, en su 
desarrollo, con la aparición e introducción de las TIC en los centros docentes tal realidad va cambiando día a día, tendiendo a desaparecer (Marín, Ramírez y Sampedro, 2011: 110), de ahí que La investigación llevada a cabo en 2006 Wighting, y refutada por Wilson en 2014, ya ponía de manifiesto el aprendizaje significativo se produce con más efecto en aquellas aulas en las que se emplean las metodologías digitales frente a las que continúan utilizando las tradicionales. Como sostienen Ricoy y Fernández (2013) y Ozkan (2015), el rápido auge de las TIC está provocando que el número de oportunidades de aprendizaje se multipliquen y se produzca una renovación didáctica, y es ahí donde los videojuegos juegan un papel importante.

Como ya señalaban Díez (2007) y Granek, Gorbet y Sergio (2010) el empleo de los videojuegos permite trabajar para que aumente la capacidad de coordinación, la percepción espacial y se pueda ampliar el campo visual, elementos que avalan pues la propuesta aquí presentada así como los procesos de socialización.

No obstante, compartimos con Del Moral, Villalustre, Yuste y Esnaola (2012) y Gros (2014), la necesidad de planificar su uso en el aula, dado que su empleo de manera irreflexiva y no planificada puede pervertir su utilización como recurso metodológico. En este sentido se abre una línea de trabajo al hilo de esta propuesta, que no es otra que la formación de los docentes en el uso de este tipo de recursos, ante los que no todos están familiarizados.

\section{REFERENCIAS}

aDeSe

(2012).

Anuario. http://www.adese.es/anuario2012/ANUARIO_ADESE 2012.pdf (19/11/2015)

Bidarian, S., Bidarian, S., y Davoudi, A. M. (2011). A model for application of ITC in the process of teaching and learning. Procedia Social and Behavioral Sciences, 29, 1032-1041. doi:10.1016/j.sbspro.2011.11.336

Blumberg, F.C. y Randall, J. D. (2015). What do children and adolescents say they do during videogame play? Journal of Applied Developmental Psychology, 34, 82-88. Doi: 10.1016/j.appdev.2012.11.004 (19/11/2015).

Cabero, J. (2009). Educación 2.0. ¿Marca, moda o nueva visión de la educación? En C. Castaño (coord.). Web 2.0. El uso de la web en la sociedad del conocimiento. Investigaciones e implicaciones educativas. (pp. pp. 930). Venezuela: Universidad Metropolitana.

Contreras, R. (2013). Acercamiento a las características de los videojuegos y sus beneficios en el aprendizaje. II Congreso Internacional de Videojuegos y Educación,Cáceres. $\quad$ pp 387-394. http://repositori.uvic.cat/bitstream/handle/10854/2753/ar tconlli_a2013_contreras_ruth_acercamiento_caracteristi cas_videojuegos.pdf?sequence $=1(19 / 11 / 2015)$.

Cuenca, J. Mª, Martín, M. J. y Estepa, M.J. (2014). El uso de los videojuegos en la formación inicial del profesorado de educación infantil para la enseñanza de las Ciencias Sociales. En J. Pagán y A. Santiesteban (eds.). Una mirada al pasado y un proyecto de futuro. Investigaciones e innovación en Didáctica de las Ciencias Sociales. (pp. 429-438). AUPDCS: Barcelona.

Danciu, E. y Grosseck, G. (2011). Social aspects of web 2.0 technologies: teaching or teachers' challenges? Procedia Social and Behavioral Sciences, 15. 3768-3773. DOI: 10.1016/j.sbspro.2011.04.371

Del Moral, E., Villalustre, L., Yuste, R. y Esnaola, G. (2012). Evaluación y diseño de videojuegos: generando objetos de aprendizaje en comunidades de práctica. RED, Revista de Educación, 33, 1-17. http://sociales.redalyc.org/articulo.oa?id=54724495002 (19/11/2015).

Díez, E.J. (2007). El género de la violencia en los videojuegos y el papel de la escuela. Revista de Educación, 342, 127 146. http://www.mecd.gob.es/dctm/revista-deeducacion/articulosre342/re34207.pdf?documentId=090 $1 \mathrm{e} 72 \mathrm{~b} 8123 \mathrm{cff5}(19 / 11 / 2015)$.

Engelhardt, C. R. y Mazurek, M. O. (2014). Video game access, parental rules, and problem behaviour: A study of boys with autism spectrum disorder. Autism, 18(5), 529-537. Doi: $10.1177 / 1362361313482053$.

Fandos, M. (2006). El reto del cambio educativo: nuevos escenarios y modalidades de formación. Educar, 38, 243-258.

García, R., Cortés, S. y Lacasa, P. (2014). Audiencias creativas y diseño de videojuegos. Revista de Estudios de la Juventud, 106, 133-148.

Granek, J.A., Gorbet, D.J. y Sergio, L.E. (2010). Extensive video-games experience afters cortical networks for complex visuomotor transformations. Cortex, 46(9), 1165-1177. Doi: 10.1016/j.cortex.2009.10.009.

Gros, B. (2014). Análisis de las presentaciones de los juegos digitales para la docencia universitaria. Revista Interuniversitaria de Formación del Profesorado, 79, $116-128$.

Gutiérrez, A. (2008). Las TIC en la formación del maestro "realfabetización" digital del profesorado. Revista Interuniversitaria de Formación del Profesorado, 63 (22,3), 191-206.

Kebritchi, M., Hirum, A. y Bai, H. (2010). The effects of modern mathematics computer games on mathematics achievement and class motivation. Computer $y$ Education, 55(2), 427-443. DOI 10.1016/j.compedu.2010.02.007.

Laru, J.; Näykki, P. y Jävelä, S. (2012). Supporting small-group learning using multiple web 2.0 tools: a case study in the higher education context. Internet and Higher Education, 15, 29-38 doi. 10.1016.jhedu.2011.08.004.

Ley Orgánica para la Mejora de la Calidad Educativa (2013). http://www.mecd.gob.es/servicios-al-ciudadanomecd/participacion-publica/lomce.html. (19/11/2015).

Marín, V. (2012a). El ayer y hoy de los videojuegos. En V. Marín (coord.). Los videojuegos y los juegos digitales como materiales educativos. (pp. 19-33). Madrid: Síntesis.

Marín, V. (2012b). Investigando sobre el potencial psicosocioeducativo de los videojuegos y juegos digitales. En V. Marín (coord.). Los videojuegos y juegos digitales como materiales educativos. (pp.193218). Síntesis: Madrid.

Marín, V. (2014). Aprendiendo a través de los videojuegos. La opinión de los y las jóvenes educadores y educadoras. Revista de Estudios de Juventud, 106, 165-149. http://www.injuve.es/sites/default/files/2014/47/publicac 
iones/10\%20Aprendi\%C3\%A9ndo\%20a\%20trav\%C3\% A9s\%20de\%20los\%20videojuegos.pdf (19/11/2015).

Marín, V. (2015). La gamificación educativa. Una alternativa para la enseñanza creatividad, Digital Educational Review, 27, 1-4.

Marín, V., Ramírez, A. y Sampedro, B. (2011). Moodle y estudiantes universitarios. Dos nuevas realidades del EEES. Profesorado, Revista de Currículum y formación del Profesorado, 15(1), 109-120.

Marín, V., López, M., y Maldonado, G. A. (2015). Can gamification be introduced within primary classes? Digital Educational Review, 27, 53-66.

Marqués, P. (2007). Competencias básicas en la sociedad de la información.

http://peremarques.pangea.org/competen.htm (19/11/2015).

Martin, M. (2015). Videojuegos y aprendizaje colaborativo. Experiencias en torno a la etapa de Educación Primaria. Enseñanza y Teaching, 16(2), 69-89.Doi: http://dx.doi.org/10.14201/eks20151626989 (19/11/2015).

Moncada, J. y Chacón, Y. (2012). El efecto de los videojuegos en variables sociales, psicológicas y fisiológicas en niños y adolescentes, RETOS, Nuevas Tendencias en Educación Física, Deporte y Recreaciones, 21, 45-49.

Munyoka,W., Kadymatimba, A., Manzirra,M. F., Tutoni,D., ChibondaT., y Madzwamuse, S. (2014). Promoting active learning in Higher education through the use of social media: experience of Universtiy of Venda. South Africa International Conference on Education, pp. 207216). African Academic Research Forum: South Africa.

Ozkan, M. (2015). Wikis and blogs in foreign language learning from the perspectives of learners. Procedia Social and Behavioral Sciences, 192, 672-678. Doi: 10.1016/j.sbspro.2015.06.102.

Ricoy, M M C. y Fernández, J. (2013). Contribuciones y controversias que genera el uso de las TIC en la educación superior: un estudio de caso. Revista de Educación, 360, 509-532. Doi: 10.4438/1988-592X-RE2011-360-125

Wighting, M. J. (2006). Effects of computer use on high School student's sense of community. The Jorunal of Educational Research, 99(6), 371-379.

Wilson, K. B. (2014). Impact of emerging technologies on teacher education: experiences of teacher trainees. Journal of Education and Practice, 5(28), 168-176. 